\title{
Speech timing prediction in multimodal human-computer interaction
}

\author{
Marie-Luce Bourguet \& Akio Ando \\ Nippon Hoso Kyokai (Japan Broadcasting Corporation) \\ Science and Technical Research Laboratories \\ 1-10-11 Kinuta Setagaya-ku Tokyo 157 Japan \\ bourguet@acod1.strl.nhk.or.jp, ando@strl.nhk.or.jp
}

\begin{abstract}
This paper proposes a quantitative model of natural modality integration for speech and pointing gestures. An experiment is described that study temporal synchronization between speech and pointing gestures during multimodal interaction. The end of a pointing gesture (MT) is shown to be synchronized with either the key word of an expression or the deictic marker of a deictic expression. It is also shown that MT tends to be slightly behind the beginning of its associated word; this tendency becoming more marked for lengthy expressions (more than 6 syllables). All correlation scores were found to be above 0.9 .

Automatic understanding of natural multimodal interaction may advance only through good models of spontaneous communication. Specifically, the model proposed here can be efficiently used in the speech recognition system or in the interface management system to predict speech timing according to gesture timing.
\end{abstract}

KEY WORDS Multimodal Interaction, Speech Recognition, Pointing Gestures, Synchrony, Predictive Model

\section{INTRODUCTION}

Natural communication between people and computerized systems inevitably involves voice interaction. This is because speech is the most natural and widely used human mode of communication. But speech is seldom used alone. In fact, natural communication also involves a variety of bodily movements that actively participate in the communicative process. For example, verbal communication is often accompanied by hand gestures, eyes and head movements, face expressions that fulfill divers communicative functions. These nonverbal acts usefully accompany the spoken words by adding nuance of meaning (facial expressions), maintaining good quality of communication (glance, smile), or clarifying an expression (hand signs).

Researchers in experimental psychology, have described different aspects of natural speech and gesture integration. It has been shown for example that human perception of speech is achieved via a variety of bottomup and top-down sources of information. When people watch a speaker, they are influenced both by what they see and what they hear (see the McGurk effect [McGurk, 1976]). Research is currently being done on multimodal systems that integrate auditory and visual information at low-level signal processing (automatic lip-reading), for better overall speech recognition.

But natural communication is much more than just speech and lip-reading. [Rime, Schiaratura, Hupet \& Ghysselinckx, 1984] have shown that verbal encoding was not dissociable from body movement. In an experiment, they immobilized the head, arms, hands, legs and feet of subjects during a conversation, and observed the effect of immobilization on the content of speech. Not only a significant decrease for vividness of imagery in the dialogues was found, but a significant 
increase in the activity of the body zones that remained free to move (the eyebrows, eyes, mouth and fingers) was also noted. These data suggest that the gestural motor activity of a speaking person is inextricably linked to his or her verbal encoding activities.

One of the most visible manifestations of speechrelated non verbal behavior is hand gesturing. Various classifications have been proposed for hand movements that occur with speech [Freedman 1972, Cosnier 1982, McNeill 1985] although all of them rely on a system proposed earlier by [Efron, 1941/1972]. The [Rime \& Schiaratura, 1991] "revised Efron system of gestures" distinguishes four types of hand gestures within the broader category of "depictive gestures" (gestures that refer to the object of the speech):

1. iconic gestures used by a speaker to display information about the shape of objects, spatial relations and actions.

2. pantomimic gestures involving the manipulation of some invisible object or tool in contact with the speaker's hand.

3. symbolic gestures that can be translated directly to some verbal meaning (e.g. the "OK" posture made with the fingers).

4. deictic gestures such as pointing to direct the listener's attention to objects or events in the surrounding environment.

With regard to temporal synchronization between speech and gestures, several studies suggest that gestures usually precede speech. [Dittman \& Llewellyn, 1969] observed that hand movements were particularly concentrated around the first word of utterances. [Kendon, 1972] concludes from a speech sample analysis that when a bodily movement is precisely related to a word in a sentence, the movement in question begins before the corresponding word. Finally, [Butterworth \& Beattie, 1978] conclude from similar analyses, that the initiation of iconic gestures usually precedes the word with which they are associated.

All models that have been proposed so far, to describe the temporal relationships between speech and gestures, are essentially of a qualitative nature. They can not be easily used in the human-computer interface. In contrast, the goal of our research is to begin specifying quantitative models of temporal synchronization between speech and gestures, to guide the design of robust multimodal systems.
In the following sections, we briefly review the difficulties inherent to speech and gesture integration in the human-computer interface. Then an experiment that was designed to study how people integrate their use of spoken and gesture input is described. It is followed by a discussion on the application of a predictive model of speech and gesture integration in the interface management system and in the speech recognition system.

\section{SPEECH AND GESTURE INTEGRATION IN THE HUMAN- COMPUTER INTERFACE}

The technology of automatic speech recognition has advanced to the point where rudimentary conversational interaction can be reliably accomplished for welldelimited tasks. Also, systems that can isolate and track a user's body, recognize gestures performed by his/her hands and face are currently under experimentation. These technologies, speech recognition along with computer vision, although still imperfect, have developed enough that it is critical to address now the question of their integration in the human-computer interface.

This is not an easy task however. Speech recognizers are still prone to recognition errors, which is particularly true in noisy environments and for degraded auditory inputs. We are forced to note that even after decades of effort, speech recognition by machine remains far inferior to human performance. Position trackers also show inaccuracies. Moreover, with these new devices (speech recognizers, eye trackers, body trackers, etc.), the user is set free to behave and communicate with the machine in a natural and spontaneous way; but on the other hand, it becomes increasingly difficult for the interface management system to determine when input has occurred and the precise value of that input.

Even the integration of speech and rather easily recognizable pointing gestures is not a straightforward operation. As shown in the previous section, the use of speech and gestures by human beings, is fully integrated and coordinated. But unfortunately, the devices used to interact with computers have not been designed to cooperate. The flexibility of interaction they offer is not addressed appropriately by current software tools. 
Nevertheless, the integration of speech and pointing gestures in the human-computer interface has already been implemented in several experimental systems. Most of these systems use pointing to help prevent ambiguity in a recognized speech utterance. Pointing, associated with speech, can provide additional information about the user's intentions through phenomena of redundancy, disambiguation or complementarity. This additional information is successfully exploited at the semantic or pragmatic levels of analysis.

Our approach to the problem is somewhat different but complementary. We believe that, to the extent that human behavior can be quantified, communicative behavior models are necessary to perform a good integration of audio and visual information in the interface management system. The following section describes a small scale experiment designed to begin specifying how people integrate their use of pointing gestures and spoken input during multimodal humancomputer interaction. Our assumption is that speech occurrence time can be predicted according to pointing gesture timing.

\section{METHOD}

\subsection{Subjects, tasks and procedure}

Twelve subjects, presenting various degrees of familiarity with computers, participated as unpaid volunteers in the experiment. All subjects were righthanded and native speakers of Japanese.

The experiment was divided in three tasks involving increasing semantic efforts as well as increasing levels of freedom in the use of gestures and speech.

For the first task (the "single word task"), subjects were shown on the computer screen sets of square figures of various sizes and colors. They were instructed to point at each figure while uttering the figure's color. Pointing gestures had to be performed with the right hand, starting from a fixed location on the desk, and ending with one finger touching the computer screen. After each gesture, the hand had to be brought back onto the desk. Subjects could move on the next figure at their own pace, but the pointing gesture (movement between the desk and the computer screen) had to be executed as quickly as possible. Possible colors were: "ao" (blue), "midori" (green), "murasaki" (purple) and "nezumi iro" (gray).

For the second task (the "question/answer task"), subjects were shown on the computer screen sets of pictures representing various musical instruments, or sports, or animals, or Japanese dishes, etc. Subjects were then asked questions related to the pictures such as "Which dish is made of rice and fish?", "Which sport is practiced on the snow?", etc. Each question had to be answered using unconstrained speech and simultaneous pointing gesture. Gestures had to be executed as for the previous task except that no speed constraint was imposed.

For the third task (the "drawing task"), subjects were asked to manipulate computer objects in order to build consistent drawings. A model to be reproduced as well as the constituent parts of the model were simultaneously provided on the computer screen (see an example on Figure 1). Objects' manipulation could be done by ordering commands to the computer ("Put the red triangle here", "Remove that", etc.) using speech and pointing gestures. No constraint was imposed on the use of speech and gestures. An operator, acting on a duplicated display, was actually monitoring the drawing editor. Subjects were aware of the operator's job and were asked to speak and act as naturally as possible.

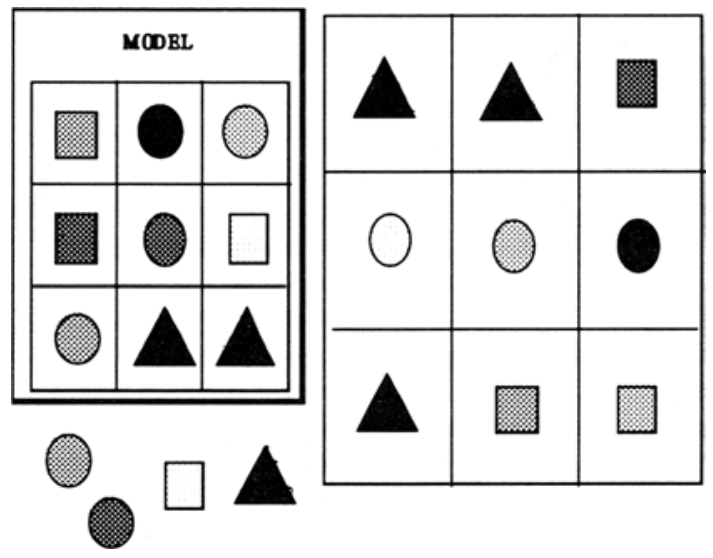

Figure 1: Example of a "drawing task". Subjects are asked to manipulate the graphical objects, using spoken commands and pointing gestures, to produce a drawing similar to the model shown on the left side of the figure. 


\subsection{Data collection}

The three tasks, performed by the twelve subjects, were videotaped. The relative timing of speech and pointing gestures was then carefully measured in terms of video frames ( 30 frames per second).

For the first and second task, a "multimodal record" is made of one utterance and its associated pointing gesture. A new time reference is calculated for each multimodal record and corresponds to the initiation of the gesture (when the hand quit the desk). The end of a gesture, which also corresponds to the movement duration (or movement time: MT), is calculated when the finger reach the computer screen (Figure 2).

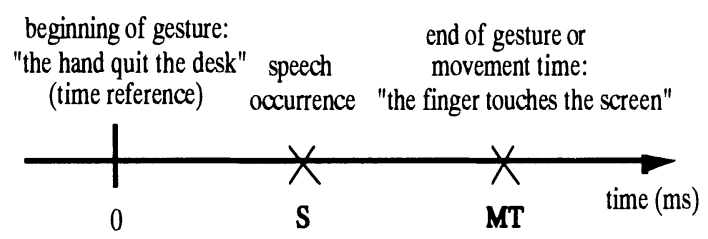

Figure 2: Calculation of movement time.

Speech occurrence time (S) is calculated according to the type of utterance. For a single word (first task), $\mathrm{S}$ corresponds to the beginning of the first syllable of the word. For deictic expressions, S corresponds to the beginning of the first syllable of the deictic marker ("kore", "kono" (this), "sore", "sono" (that), "koko" (here), etc.). For the other types of utterance, S corresponds to the beginning of the first syllable of the most significant word (keyword). This way of calculating speech occurrence will be discussed later.

For the third task, timing is measured in a similar way, except for compound commands which are made of two gestures (e.g. "kore ha, koko ni oite kudasai" (put this here)). In that case, the time reference is calculated at the beginning of the first gesture and used as such for the whole command thus for two consecutive multimodal records (Figure 3 ).

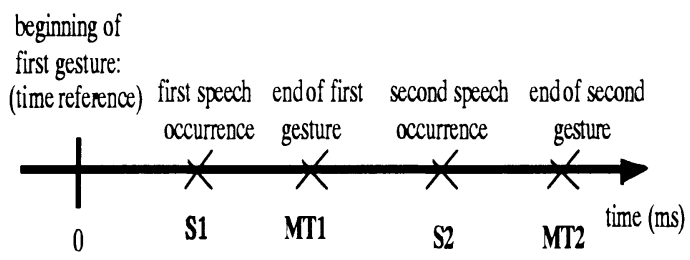

Figure 3: Speech occurrence time and movement time calculation for complex commands.

\section{RESULTS}

\subsection{Using the Fitts" law ("single word task")}

The Fitts' law [Fitts, 1954; MacKenzie, 1992 for a review of the Fitts' law) is used in $\mathrm{HCI}$ to measure and predict movement time for tasks such as text or object selection with a pointing device. The Fitts' law states that a movement tasks' difficulty (ID, the "index of difficulty") can be quantified using information theory by the metric "bits". Specifically,

$$
\text { ID }=\log _{2}(2 \mathrm{~A} / \mathrm{W})
$$

where $\mathrm{A}$ is the distance or amplitude to move and $\mathrm{W}$ the width or tolerance of the region within which the move terminates. From Equation 1, the time to complete a movement task is predicted using a simple linear equation, where movement time (MT) is a linear function of ID,

$$
\mathrm{MT}=\mathrm{a}+\mathrm{b} \text { ID }
$$

In a Fitts' law experiment, multiple trials are conducted for each task condition (A, W), then measurements are generally aggregated across subjects resulting in one data point for each task condition.

During the first task of our experiment we collected a total of 1440 trials. Each square figure was characterized by an index of difficulty ranging from 2.5 to 7 (for a total of 10 different IDs), as well as a color (among the 4 possible colors). 40 different task conditions were thus defined, and for each task condition 36 trials were collected $(1440 /(10 * 4)=36)$. Data is represented in Figure 4. After applying tests of correlation and linear regression, we found that movement time (ms) could be predicted as:

$\mathrm{MT}=345+36 \mathrm{ID}$

with a very high correlation score of $r=0.99$. 


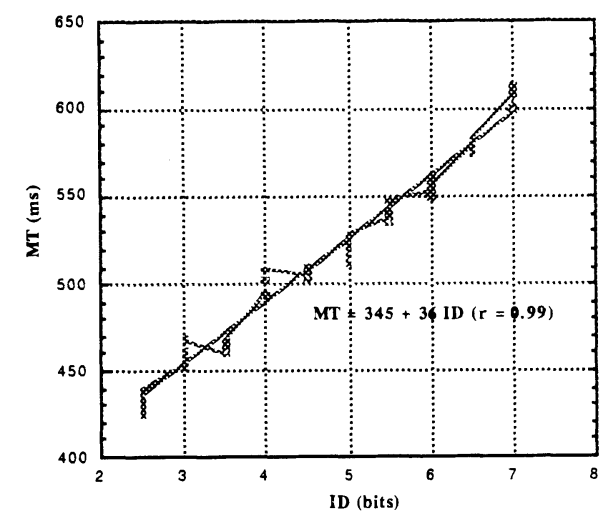

Figure 4: Plot and regression line for $M T=f(I D)$ (first task). Each data point is the mean of 36 trials. There is a strong correlation between MT and ID (Fitts' law).

The next step of our research was to relate speech occurrence time to movement time. First, the effect of the length of speech (total number of syllables) on movement time was examined. Correlation and linear regression tests were applied again separately for each color variable. Let's recall here that the four possible colors were "ao" ( 2 syllables), "midori" ( 3 syllables), "murasaki" (4 syllables) and "nezumi iro" (5 syllables). The four equations of regression line that were found are as follows:

$$
\begin{array}{ll}
\text { ao: } & \mathrm{MT}=334+38 \mathrm{ID}(\mathrm{r}=0.994) \\
\text { midori: } & \mathrm{MT}=345+35 \mathrm{ID}(\mathrm{r}=0.994) \\
\text { murasaki: } & \mathrm{MT}=343+37 \mathrm{ID}(\mathrm{r}=0.991) \\
\text { nezumi iro: } & \mathrm{MT}=357+34 \mathrm{ID}(\mathrm{r}=0.99)
\end{array}
$$

Equations 3, 4, 5, 6 and 7 are very similar (time differences are smaller than measurement precision: 1 video frame $=33.33 \mathrm{~ms}$ ), which let us suppose that the length of speech had no sensitive effect on movement time during the first task.

Second, the effect of movement tasks' difficulty (ID) on time delay between MT and S (D = MT - S) was studied (Figure 5). A low correlation score was found between $D$ and ID $(r=0.202)$. For ID ranging from 2.5 to $7, \mathrm{D}$ is always positive. In other words, $\mathrm{S}$ always precedes MT.

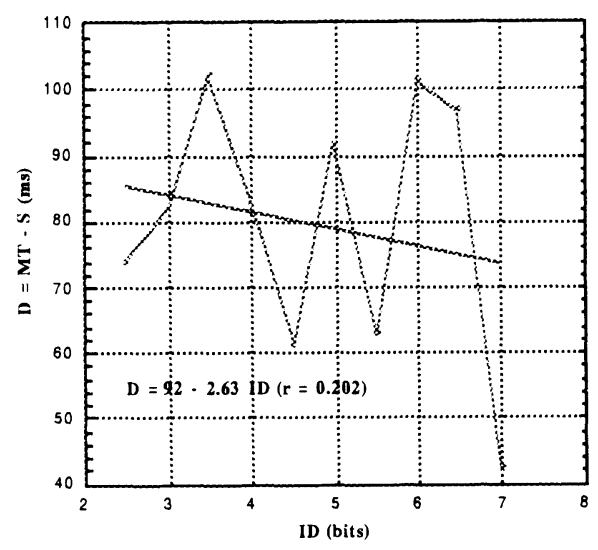

Figure 5: Plot and regression line for $D=f(I D)$ (first task). Each data point is the mean of 144 trials.

Next, the effect of the length of speech (n) on D was examined (Figure 6). Again, linear regression test was applied and a very strong correlation score was found between $\mathrm{n}$ and $\mathrm{D}$ :

$$
D=37+12 n(r=0.962)
$$

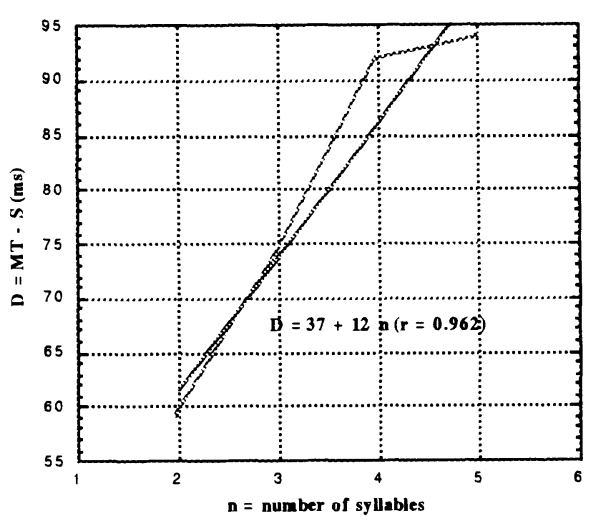

Figure 6: Plot and regression line for $D=f(n)$ (first task). Each data point is the mean of 360 trials. 
From Equation 8, it can be seen that $\mathrm{D}$ increases with $\mathrm{n}$, thus that the advance of $\mathrm{S}$ on MT increases with $\mathrm{n}$. This means that the end of gesture (MT) doesn't always coincide with the beginning of the first syllable but tends to coincide with a syllable of more central position in the word. The calculation of $D$ for $n$ varying from 2 to 5 , gives the following values $(\mathrm{ms}): 61(\mathrm{n}=2), 73(\mathrm{n}$ $=3), 85(n=4)$ and $97(n=5)$. On average, the length of a syllable is about $100 \mathrm{~ms}$, thus MT coincide with the first syllable for $1<\mathrm{n}<6$. For $n$ varying from 6 to 14, MT coincides with the second syllable, etc.

Finally, tests of correlation and linear regression were applied again as shown in Figure 7 to study the relationship between $\mathrm{S}$ and $\mathrm{MT}$. The following equation of regression line was found:

$$
\mathrm{S}=-128+1.15 \mathrm{MT}(\mathrm{r}=0.979)
$$

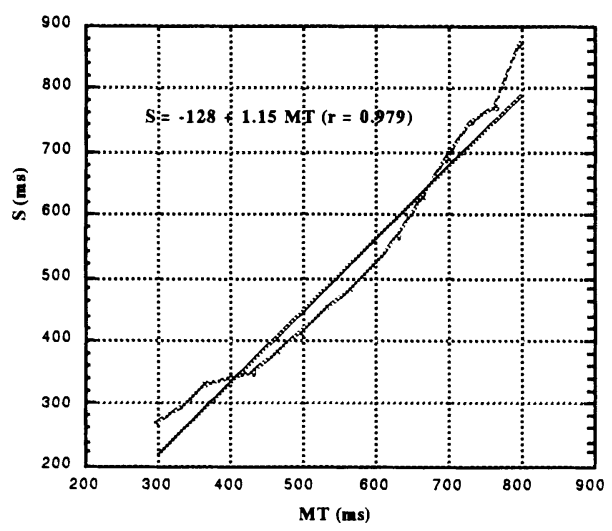

Figure 7: Plot and regression line for $S=f(M T)$ (first task). Each data point is the mean of several trials (from 1 trial to 269 trials) for a total of 1440 trials.

A very high correlation score was found between $S$ and MT. Equation 9 confirms the observation that $S$ tends to precede MT. However, the tendency is inverted for MT $\geq 853.33 \mathrm{~ms}$ (out of data range). For MT that ranges from 300 to $800 \mathrm{~ms}, \mathrm{~S}$ varies from 217 to 792 $\mathrm{ms}$ and D from 8 to $83 \mathrm{~ms}$. MT always coincide with the first syllable of the word.

\subsection{Speech timing prediction (second and third tasks)}

During the second and third tasks, no constraint on the use of speech and gestures was imposed. MT was observed to be synchronized with either the deictic marker of a deictic expression, or the key word of other expressions (see previous section "data collection").

For the data collected during the second task (the "question/answer task") the following equation was calculated:

$$
\mathrm{S}=-184+0.97 \mathrm{MT}(\mathrm{r}=0.929)
$$

Each data point was the mean of several trials (from 1 to 38 trials) for a total of 420 trials.

For the data collected during the third task (the "drawing task"), the following equation was calculated:

$$
\mathrm{S}=-80+0.94 \mathrm{MT}(\mathrm{r}=0.967)
$$

Each data point was the mean of several trials (from 1 to 35 trials) for a total of 734 trials.

Equations 10 and 11 are very similar. As expected, natural integration of speech and pointing gestures is task independent. Unlike what is suggested by Equation 9 (first task), D tends to increase with movement time. Within the range of the collected data $(300<\mathrm{MT}<$ 5000 ), MT is found to take place between the first and the fourth syllable of either the key word or the deictic marker (or the first syllable of the following word). This can be explained in two ways. Longer gestures actually correspond to:

(1) hesitation, movement time is then delayed;

(2) the second part of a complex command ("drawing task"). If speech is short, the gesture is slightly behind (e.g. "kore ha, koko desu" (this is here) + two pointing gestures).

\section{DISCUSSION}

The results of our experiment are in accordance with previous findings on temporal synchronization between speech and gestures (see the Introduction of this paper). In particular, [Kendon, 1972] says that bodily movements, when precisely related to a word in a sentence, begin before the word in question. We found that hand pointing gestures always start before their associated spoken word (the key word or the deictic marker of an expression), and that they terminate in synchronization with (or slightly behind) the beginning of the word. 
Such a quantitative model of natural speech and gesture integration can be used in both the speech recognition system and the interface management system.

In automatic speech recognition (ASR), errors are typically divided into three categories: substitution (misrecognition of a word), insertion (abusive recognition of noise or unintentional utterance) and false rejection (non-recognition of a word). The correct association of speech and gesture, according to a predictive model, could prevent an erroneous rejection decision. Similarly, in case of bad association of speech and gesture, insertion error could be avoided. With regard to substitution errors, one of the main difficulty in ASR, is word boundary detection, especially in noisy environment. A quantitative model of natural modality integration that provides information on speech timing (beginning time of a word in an utterance) could help solving the problem.

In the interface management system, it is often difficult to determine which input is related to which input and if two inputs are actually related. Alternate usage of concurrent multimodality and compound multimodality makes things even more difficult [Bourguet, 1992] (Figure 8).
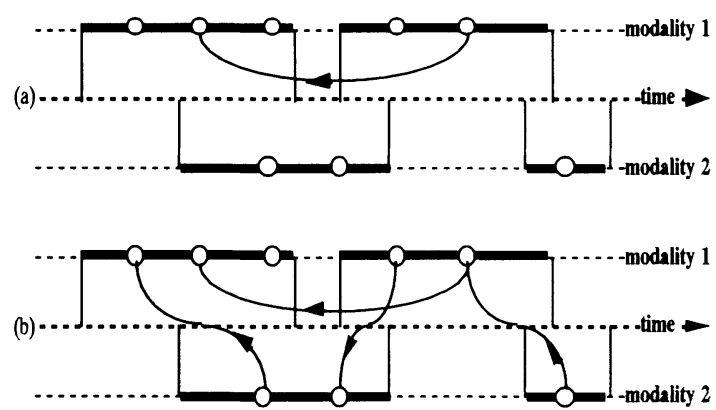

Figure 8. Concurrent multimodality (a) allows a system user to simultaneously input two independent commands by means of two different modalities. Information merging (symbolized by an arrow), if needed, is unimodal (e.g. anaphora resolution in natural language). Compound multimodality (b) allows a single command to be given by means of two or more modalities. Information merging is made across modalities.
The choice of good criteria to guide information fusion is of great importance. In most current multimodal systems, information merging is done at a high level of abstraction (semantic or pragmatic level), which minimizes the risks of erroneous fusion. Such fusion mechanisms are certainly robust but generally application-dependent. Conversely, information fusion based on temporal criteria is currently less reliable but allows the design of more generic multimodal interface tools. This lack of reliability is due to the lack of good quantitative models of natural modality integration.

\section{CONCLUSION}

Our assumption, prior to the experiment, was that speech timing could be predicted according to pointing gesture timing. The results of the experiment suggest that it is true. The predictive equations that were calculated on the different sets of data (Equations 9, 10 and 11) actually show that speech occurrence time and movement time are closely related. All correlation scores were found to be above 0.9 (correlations above 0.9 are considered very high for any experiment involving measurements on human subjects). The end of a pointing gesture (MT) has been shown to be synchronized with either the key word of an expression or the deictic marker of a deictic expression. It has also been shown that MT tended to be slightly behind the beginning of its associated word; this tendency becoming more marked for lengthy expressions (more than 6 syllables).

The model that was proposed here is based on a small set of data and needs to be refined. With more data, it would be possible to closely examine the relative timing of speech and gestures in many different circumstances and for different types of utterances. This is part of our future work.

We chose to study speech timing according to gesture timing because in the case of speech and hand pointing gestures (that can be easily recognized using a touch panel), most of the inaccuracy lies in the speech recognition process. However, for more complex gestures, or for pointing gestures performed at distance, a similar model could be calculated to predict gesture timing according to speech timing. 


\section{ACKNOWLEDGEMENTS}

Many thanks to Mr. Takehiro Izumi, Executive Research Engineer (and former Director General) at NHK Science and Technical Research Laboratories; and to Mr. Taiji Nishizawa, Director General of NHK Science and Technical Research Laboratories for inviting me in their laboratory. Special thanks to Dr. Eiichi Miyasaka, Director of the Auditory Science \& Acoustics Research Division at NHK Science and Technical Research Laboratories for supporting this research.

\section{REFERENCES}

Bourguet, M.L. (1992). Conception et realisation d'une interface de dialogue personne-machine multimodale. $\mathrm{PhD}$ dissertation, Institut National Polytechnique de Grenoble, France.

Butterworth, B. \& Beattie, G. (1978). Gesture and silence as indicators of planning in speech. In R.N. Campbell \& P.T. Smith (Eds.), Recent advances in the psychology of language, 347-360. New York: Plenum.

Cosnier, J. (1982). Communications et langages gestuels. In J.Cosnier, J. Coulon, J. Berrendonner \& C. Orecchioni (Eds.), Les voies du langage: communications verbales, gestuelles et animales, 255303. Paris: Dunod.

Dittman, A.T. \& Llewellyn, L.C. (1969). Body movement and speech rhythm in social conversation. Journal of Personality and Social Psychology, 11 (2), 98-106.

Efron, D. (1941/1972). Gesture, race and culture. The Hague: Mouton.
Fitts, P.M. (1954). The information capacity of the human motor system in controlling the amplitude of movement. Journal of Experimental Psychology, 47, 381-391.

Freedman, N. (1972). The analysis of movement behavior during the clinical interview. In A.R. Siegman \& B. Pope (Eds.), Studies in dyadic communication, 153-175. Elmsford, NY: Pergamon.

Kendon, A. (1972). Some relationships between body motion and speech. In A.R. Siegman \& B. Pope (Eds.), Studies in dyadic communication, 177-210. Elmsford, NY: Pergamon.

MacKenzie, I.S. (1992). Movement time prediction in Human-Computer Interfaces. Proc. Graphics Interface' 92, Morgan Kaufmann, 140-150.

McGurk, H. \& MacDonald, J. (1976). Hearing lips and seeing voices. Nature, $264,746-748$.

McNeill, D. (1985). So you think gestures are nonverbal? Psychological Review, 92 (3), 350-371.

Rime, B., Shiaratura, L., Hupet, M. \& Ghysselinckx, A. (1984). Effects of relative immobilization on the speaker's nonverbal behavior and on the dialogue imagery level. Motivation and Emotion, 8, 311-325.

Rime, B. \& Schiaratura, L. (1991). Gesture and speech. In R.S. Feldman \& B. Rime (Eds.), Fundamentals of Nonverbal Behavior, 239-281. The Cambridge University Press. 